OPEN ACCESS

Edited by:

Phuc Huu Phan,

Vietnam National Hospital of

Pediatrics, Vietnam

Reviewed by:

Tuuli Metsvaht,

University of Tartu, Estonia

Silvia Salvatore,

University of Insubria, Italy

*Correspondence:

Yajuan Wang

cxswyj@vip.sina.com

Specialty section: This article was submitted to Neonatology,

a section of the journa

Frontiers in Pediatrics

Received: 21 February 2021 Accepted: 26 April 2021

Published: 25 May 2021

Citation:

Wu D, Ding Y, Yao K, Gao W and Wang $Y$ (2021) Antimicrobial

Resistance Analysis of Clinical Escherichia coli Isolates in Neonatal Ward. Front. Pediatr. 9:670470. doi: 10.3389/fped.2021.670470

\section{Antimicrobial Resistance Analysis of Clinical Escherichia coli Isolates in Neonatal Ward}

\author{
Dan $\mathrm{Wu}^{1}$, Yijun Ding ${ }^{1}$, Kaihu $\mathrm{Yao}^{2}$, Wei Gao ${ }^{2}$ and Yajuan Wang ${ }^{3 *}$ \\ ${ }^{1}$ Department of Neonatology, Beijing Children's Hospital, Capital Medical University, National Center for Children's Health, \\ Beijing, China, ${ }^{2}$ Laboratory of Dermatology, Beijing Pediatric Research Institute, Beijing Children's Hospital, Capital Medical \\ University, National Center for Children's Health, Beijing, China, ${ }^{3}$ Department of Neonatology, Children's Hospital, Capital \\ Institute of Pediatrics, Beijing, China
}

Background: Escherichia coli (E. coli) column for one of the most common pathogens causing neonatal infections. The emergence of antibiotic-resistant bacteria is a major cause of treatment failure in infected newborns. The purpose of this study was to describe antibiotic and multidrug resistance of $E$. coli strains isolated from neonates with infection throughout the years 2009-2011.

Methods: The antimicrobial susceptibility testing of $E$. coli strains to selected antibiotics was assessed using the E-test technique on the Mueller-Hinton agar. The antimicrobial tests included ceftazidime, cefuroxime, cefatriaxone, amoxicillin, amoxicillin-clavulanic acid, cefoperazone- sulbactam, meropenem, gentamicin, ciprofloxacin, and sulfonamides.

Results: A total of 100 E. coli strains were isolated from sputum $(n=78)$, blood $(n=10)$, cerebrospinal fluid $(n=5)$, and umbilical discharge $(n=7)$ samples of hospitalized neonates at the Beijing Children's Hospital. The highest rate of $E$. coli resistance was found in amoxicillin (85\%), followed by cefuroxime (65\%), and cefatriaxone (60\%), respectively. A total of 6 and $5 \%$ of all isolates were only resistant to amoxicillin/clavulanic acid and cefoperazone -sulbactam. The rates of resistance to ceftazidime, gentamicin, ciprofloxacin, and sulfonamides were 31, 20, 33, and 47\%, respectively. All isolates were susceptible to meropenem. Approximately $26 \%$ of all E. coli isolates were multidrug-resistant. The detection rate of ESBL-Producing E. coli was 55\%.

Conclusions: Multi-drug-resistant E. coli has become an important and complex problem in clinical treatment, and it is thus essential to monitor $E$. coli resistance in neonates.

Keywords: Escherichia coli, antimicrobial drug resistance, E-test, ESBL, neonate

\section{INTRODUCTION}

Newborns suffer high rates of mortality due to infectious diseases (1). Neonatal sepsis is the third leading cause of neonatal mortality, after prematurity and intrapartum-related complications (or birth asphyxia) (2).

Escherichia coli (E. coli) is the most common Gram-negative bacterium responsible for a variety of diseases as a result of community and hospital acquired clinically significant blood 
stream infections (BSIs), and constitutes a major cause o-f mortality from these infections at all ages. Pathogenic E. coli strains can be divided according to infection site into intestinal and extraintestinal (ExPEC). In recent years, many scholars in North America and Europe have continuously reported ExPEC with serious pathogenicity. A contemporary collection comprising 12,737 strains from pediatric patients ( $<18$ years) isolated over a 7-year period (1998-2004) from 52 sentinel hospitals in North America showed that E. coli ranks in the top 6 pediatric pathogens (3) and that ExPEC is the leading cause of infections in neonates among gram-negative bacteria $(4,5)$.

In the past few years, antibiotics helped saving a significant number of lives and reduced the illness of several million people across the world (3). However, the remarkable benefits of antimicrobials in reducing morbidity and mortality rates have been challenged by the emergence of drug resistant strains in recent years, a more prevalent problem in developing countries for a variety of reasons $(2,3)$. The emergence and rapid spread of extended-spectrum cephalosporin and carbapenem resistance in Enterobacteriaceae is becoming a global health challenge. In addition, antibiotic-resistant $E$. coli are also increasing and becoming a major threat for global human health.

The emergence of multidrug-resistant E. coli, has been observed in various countries over the past decades. The increasing resistance to cephalosporins, especially the parallel rise in the frequency of multidrug-resistant E. coli, constitutes an increasing concern for the treatment of $E$. coli disease. The predominant mechanism of resistance to $\beta$-lactam antibiotics in $E$. coli is the production of plasmid-borne extended-spectrum $\beta$-lactamases (ESBLs). Since the first report in the early 1980s, ESBL-producing organisms have become widespread throughout the world (6). The ESBL genes are frequently encoded on transferable plasmids that encode resistance genes, and the acquisition of these resistant genes by commensal or fecal isolates leads, in turn, to multidrug resistant (MDR) pathogens.

To the best of our knowledge, there are limited data regarding E. coli antibiotic susceptibility in neonatal invasive diseases worldwide, particularly in China. In this study, we aim to investigate antibiotic susceptibility and multi-drug resistance of E. coli isolates that cause neonatal infections in order to provide a basis for clinical treatment of $E$. coli infections.

\section{MATERIALS AND METHODS}

\section{Study Design}

This study was performed at the Beijing Children's Hospital, a tertiary facility with 110 beds in the neonatal unit, which handles more than 3,000 inpatient neonates per year. sputum, blood and/or cerebrospinal fluid (CSF) samples were taken from inpatient neonates diagnosed with pneumonia, sepsis and/or meningitis. Patients aged $<28$ days with $E$. coli positive cultures were enrolled, other bacteria strains were excluded. The study period spanned the years 2009-2011. This study was approved

Abbreviations: E. coli, Escherichia coli; ESBL, Extended-spectrum beta-lactamase; NICU, Neonatal intensive care unit; ExPEC, Extraintestinal pathogenic E. coli; CSF, Cerebrospinal fluid. by the ethics committee of Beijing Children's Hospital, in accordance with the Declaration of Helsinki. The experimental procedure is shown in Figure 1.

\section{Bacterial Identification}

E. coli species identification was performed using an ATB automatic bacterial identification instrument (France merrier company), a VITEK automatic biological analysis system (Biomerier China company) or a French merieres Merieux API system.

\section{Antimicrobial Susceptibility Testing of $E$. coli and Detection of ESBLs}

The antimicrobial susceptibility testing of $E$. coli strains to selected antibiotics was assessed with the E-test technique (AB Biodisk-solana, Sweden) on the Mueller-Hinton agar (Becton Dickinson). The antimicrobial tests included were ceftazidime, cefuroxime, cefatriaxone, amoxicillin, amoxicillin-clavulanic acid, cefoperazone-sulbactam, meropenem, gentamicin, ciprofloxacin, and sulfonamides. The minimal inhibitory concentration (MIC) values of the antimicrobial agents selected for this study were determined by an agar dilution technique on Mueller-Hinton agar (Oxoid) according to the Clinical and Laboratory Standards Institute (CLSI) recommendations (7). The E. coli strain ATCC 25922 was used for routine qualitycontrol assays. Multi-drug resistant (MDR) E. coli was defined as a strain showing non-susceptibility to at least one agent in three or more antimicrobial categories.

\section{Detection of ESBLs}

The MICs of oxyimino- $\beta$-lactams and clavulanic acid were determined at a fixed concentration of $4 \mathrm{mg} / \mathrm{l}$. The production of $E$. coli Extended-spectrum beta-lactamases (ESBLs) was determined using the double-disk synergy test (DDST). Specifically, this was performed with cefotaxime $(30 \mu \mathrm{g})$ and ceftazidime $(30 \mu \mathrm{g})$ disks placed at a distance of $20 \mathrm{~mm}$ (center to center) from the amoxicillin-clavulanic acid disk $(20 / 10 \mu \mathrm{g})$. Moreover, cefpodoxime $(10 \mu \mathrm{g})$, and aztreonam $(30 \mu \mathrm{g})$ disks were added to increase the sensitivity of the DDST. A cefepime $(30 \mu \mathrm{g})$ disk was placed in the same culture medium in order to improve the detection of ESBL during the simultaneous stable hyperproduction of an AmpC beta-lactamase. The test result was considered positive when an enhancement of the inhibition zone around at least one of the antibiotic disks (cefotaxime, ceftazidime, cefpodoxime, aztreonam, or cefepime) toward the clavulanic acid disk was observed. The control strains Klebsiella pneumoniae ATCC 700603 (ESBL positive) and E. coli ATCC 25922 (ESBL negative) were used for quality control.

\section{Statistical Analysis}

All data was prepared and analyzed with the software WHONET 5.3, which is recommended by the World Health Organization. The $X^{2}$ test was performed for comparing antibiotic and multidrug resistance proportions of E. coli strains using the SPSS version 13.0 software. Differences with a $X^{2} P<0.05$ were considered statistically significant. 


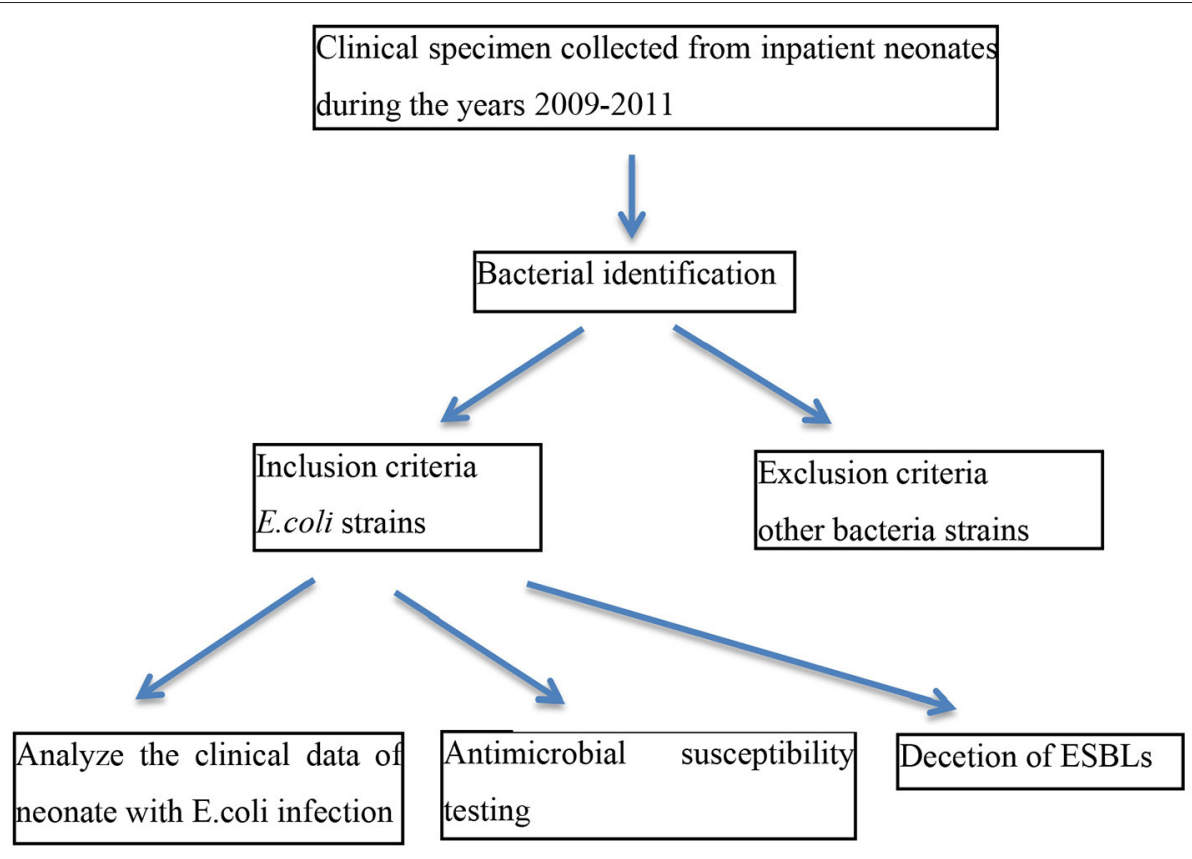

FIGURE 1 | Experimental procedure. The experimental procedure included inclusion and exclusion criteria was shown.

\section{RESULTS}

\section{Characteristics of E. coli Strains}

A total of 100 E. coli strains were collected from January 2009 to December 2011 from neonates hospitalized at Beijing Children's Hospital. The clinical information of the neonates (including age and gender) from whom $E$. coli strains were isolated, as well as the source of the isolates in the present study, are reported in Table $\mathbf{1 .}$ A total of $78 \%$ of the strains were isolated from sputum samples, $10 \%$ from blood samples, $5 \%$ from CSF samples, and $7 \%$ from umbilical discharge.

\section{Analysis of the Antimicrobial Susceptibility}

The susceptibility of the E. coli strains to 10 antibiotics and the MICs of 100 E. coli isolates are presented in Table 1. Based on the CLSI 2016 criteria, the highest resistance rate of E. coli was to amoxicillin (85\%), followed by cefuroxime (65\%), and cefatriaxone $(60 \%)$. Moreover, 6 and $5 \%$ of all isolates were resistant to amoxicillin/clavulanic acid and cefoperazonesulbactam, respectively. The resistance rates to ceftazidime, gentamicin, ciprofloxacin, and sulfonamides were 31, 20,33, and $47 \%$, respectively. All isolates were susceptible to meropenem. More details about antimicrobial resistance rates are presented in Table 2.

\section{Multidrug-Resistant $E$. coli}

The antibiotic resistance pattern of $100 \mathrm{E}$. coli isolates is shown in Table 3. Amoxicillin, cefuroxime, cefatriaxone, ceftazidime, amoxicillin-clavulanic acid, cefoperazone-sulbactam, and meropenem were classified as $\beta$-lactams. In contrast, gentamicin was classified as an aminoglycoside, while ciprofloxacin was regarded as a quinolone. We defined multidrug resistance in
E. coli as resistance to at least three distinct antibiotic families and estimated this rate at $\sim 26 \%(26 / 100)$ across all $E$. coli isolates.

Overall, the detection rate of ESBL-Producing E. coli was $55 \%$. Specifically, this rate was significantly higher $(P<0.05)$ in sputum isolates $(65 \%, 51 / 78)$ compared to aseptic humoral $(27 \%, 4 / 15)$.

\section{Inspection Situation of ESBLs}

The detection rate of ESBL-Producing E. coli was 55\%. The rate in $E$. coli isolates from sputum $(65 \%, 51 / 78)$ was higher than aseptic humoral $(27 \%, 4 / 15)$. The difference was statistically significant $(P<0.05)$.

\section{Susceptibility of ESBL-Producing $E$. coli to Antimicrobial Agents}

Importantly, the majority of the isolates were also resistant to non- $\beta$-lactam antimicrobial agents, even though the resistant rates were significantly lower than those observed in extendedspectrum $\beta$-lactamases. The differences between cefuroxime, cefatriaxone, and amoxicillin were statistically significant $(P<$ 0.05), as shown in Table 4.

\section{Clinical Treatment Condition of the Neonates With Isolated Strains in the Present Study}

The treatment process of clinical neonates is shown in Figure 2. The health conditions of all patients improved and some patients were completely cured by the end of the study. 
TABLE 1 | Clinical information of the neonates with isolated strains in the present study.

\begin{tabular}{|c|c|}
\hline Characteristics & No. of patients \\
\hline \multicolumn{2}{|l|}{ Gender } \\
\hline Male & 58 (58\%) \\
\hline Female & 42 (42\%) \\
\hline \multicolumn{2}{|c|}{ Gestational age (weeks) } \\
\hline $28-37$ & $5(5 \%)$ \\
\hline $37-42$ & 93 (93\%) \\
\hline$>42$ & $2(2 \%)$ \\
\hline \multicolumn{2}{|l|}{ Birthweight (g) } \\
\hline$<2,500$ & $2(2 \%)$ \\
\hline $2,500-4,000$ & 89 (89\%) \\
\hline$\geq 4,000$ & $9(9 \%)$ \\
\hline \multicolumn{2}{|l|}{ Post-natal age (days) } \\
\hline$<7$ & 24 (24\%) \\
\hline $7-14$ & $30(30 \%)$ \\
\hline $14-21$ & $21(21 \%)$ \\
\hline $21-28$ & 19 (19\%) \\
\hline$>28$ & $6(6 \%)$ \\
\hline \multicolumn{2}{|l|}{ Underlying diseases } \\
\hline Meningitis & $13(13 \%)$ \\
\hline Pneumonia & 87 (87\%) \\
\hline Sepsis & 27 (27\%) \\
\hline \multicolumn{2}{|l|}{ Patients' symptoms } \\
\hline Fever & 40 (40\%) \\
\hline Cough & 44 (44\%) \\
\hline Jaundice & 21 (21\%) \\
\hline Week response & $43(43 \%)$ \\
\hline Convulsion & $5(5 \%)$ \\
\hline \multicolumn{2}{|l|}{ Specimen types } \\
\hline Sputum & 78 (78\%) \\
\hline Venous blood & 10 (10\%) \\
\hline Cerebroapinal fluid & $5(5 \%)$ \\
\hline Umbilical discharge & 7 (7\%) \\
\hline \multicolumn{2}{|l|}{ Outcome } \\
\hline Cure & $88(88 \%)$ \\
\hline Improve & $12(12 \%)$ \\
\hline Unhealed & 0 \\
\hline
\end{tabular}

\section{DISCUSSION}

Escherichia coli is the most frequent Gram-negative organism causing neonatal bacteremia and sepsis (8). Among febrile infants younger than 28 days-old, the prevalence of bacteremia and meningitis is high and most commonly caused by E. coli (9). The incidence of $E$. coli early-onset sepsis in very low birth weight infants was estimated at $1.04 \%$, reaching a mortality rate of $35.3 \%$ (10). A recent meta-analysis based on a systematic review of published studies in the Chinese literature demonstrated that, among the newborn infants hospitalized in Chinese NICUs, $\sim 50 \%$ of all $E$. coli bloodstream isolates (independently of being early or late onset) were multi-drug resistant due to extendedspectrum beta-lactamase (ESBL) production $(11,12)$.
TABLE 2 | Susceptibility and MICs of 100 E. coli isolates to 10 antibiotics.

\begin{tabular}{|c|c|c|c|c|c|c|}
\hline \multirow[t]{2}{*}{ Antibiotics } & \multicolumn{3}{|c|}{ Susceptibility } & \multicolumn{3}{|c|}{ MIC $(\mu \mathrm{g} / \mathrm{ml})$} \\
\hline & S (\%) & $\mathrm{I}(\%)$ & R (\%) & $50 \%$ & $90 \%$ & Range \\
\hline Ceftazidime & 63 & 6 & 31 & 1.5 & $>256$ & $0.016-256$ \\
\hline Cefuroxime & 35 & 0 & 65 & $>256$ & $>256$ & $0.016-256$ \\
\hline Cefatriaxone & 37 & 3 & 60 & $>32$ & $>32$ & $0.002-32$ \\
\hline Amoxicillin & 9 & 6 & 85 & $>256$ & $>256$ & $1.0->256$ \\
\hline Meropenem & 100 & 0 & 0 & 0.016 & 0.094 & $0.002-32$ \\
\hline Gentamicin & 73 & 7 & 20 & 0.75 & 24 & $0.016-256$ \\
\hline Ciprofloxacin & 67 & 0 & 33 & 0.19 & $>32$ & $0.002-32$ \\
\hline Cefoperazone-sulbactam & 77 & 18 & 5 & 3 & 16 & $0.016-256$ \\
\hline Amoxicillin-clavulanic acid & 72 & 22 & 6 & 3 & 24 & $1.5-16$ \\
\hline Sulfonamides & 53 & 0 & 47 & 0.125 & $>32$ & $0.002-32$ \\
\hline
\end{tabular}

TABLE 3 | Antibiotic resistant pattern of $100 \mathrm{E}$. coli isolates.

\begin{tabular}{llcc}
\hline $\begin{array}{l}\text { Class of } \\
\text { antibiotic }\end{array}$ & Resistance pattern & $\begin{array}{c}\text { No. of } \\
\text { isolates }\end{array}$ & $\begin{array}{c}\text { Proportion } \\
\text { of all } \\
\text { isolates }\end{array}$ \\
\hline 0 & - & 5 & $5 \%$ \\
1 & $\beta$-lactams & 26 & $26 \%$ \\
2 & $\beta$-lactams+aminoglycoside & 5 & $5 \%$ \\
& $\beta$-lactams+quinolon & 19 & $19 \%$ \\
& $\beta$-lactams+sulfonamides & 19 & $19 \%$ \\
& $\beta$-lactams+aminoglycoside+quinolon & 4 & $4 \%$ \\
& $\beta$-lactams+aminoglycoside+sulfonamides & 3 & $3 \%$ \\
& $\beta$-lactams+quinolon+sulfonamides & 16 & $16 \%$ \\
& $\beta$-lactams & 3 & $3 \%$ \\
& aminoglycoside+quinolon+sulfonamides & & \\
&
\end{tabular}

TABLE 4 | Resistance rate of ESBL-Producing and non-ESBL-Producing E. coli strains.

\begin{tabular}{lcccc}
\hline & ESBL & Non-ESBL & $\boldsymbol{X}^{2}$ & $\boldsymbol{P}$ \\
\hline Ceftazidime & $20(36.4)$ & $11(24.4)$ & 1.644 & 0.1998 \\
Cefuroxime & $51(92.7)$ & $14(31.1)$ & 41.303 & 0.00 \\
Cefatriaxone & $47(85.5)$ & $13(28.9)$ & 32.997 & 0.00 \\
Amoxicillin & $51(92.7)$ & $34(75.6)$ & 5.724 & 0.017 \\
Meropenem & 0 & 0 & - & - \\
Gentamicin & $12(21.8)$ & $13(28.9)$ & 0.66 & 0.417 \\
Ciprofloxacin & $19(34.5)$ & $14(31.1)$ & 0.09 & 0.764 \\
Cefoperazone-sulbactam & $3(5.5)$ & $3(6.7)$ & 0.064 & 0.8 \\
Amoxicillin-clavulanic acid & $5(9.1)$ & $6(13.3)$ & 0.455 & 0.5 \\
Sulfonamides & $23(41.8)$ & $23(51.1)$ & 0.86 & 0.354 \\
\hline
\end{tabular}

Emerging antibiotic resistance is currently acknowledged as one of the most significant public health problems with high mortality rates associated with multidrug-resistant bacterial infections. The selective pressures imposed by antimicrobial use, overuse and misuse are driving the gradual increase in antibiotic resistance and leading to the emergence of multidrug-resistant bacterial strains. Previously treatable bacterial infections are now 


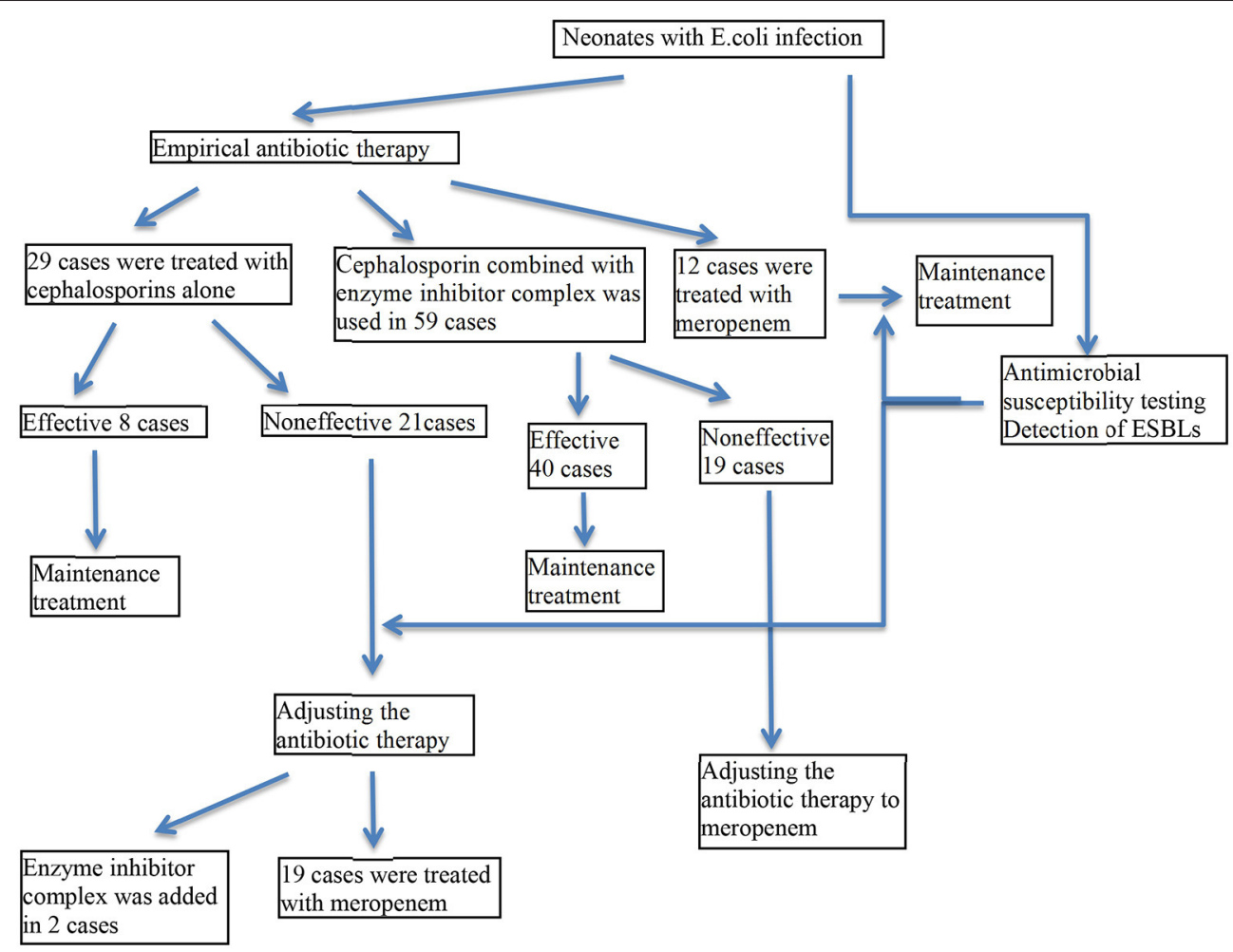

FIGURE 2 | Treatment process of clinical neonates. The treatment procedures for all clinical neonates were shown.

often untreatable or requiring the use of the last line of antibiotics (13). E. coli is the most common Gram-negative bacterial pathogen amongst resistant bacteria and causes a diverse range of diseases affecting all age groups. Multidrug-resistant, extensively drug-resistant and pan-drug-resistant strains of $E$. coli have now been reported worldwide, and this is becoming a critical global issue (14).

Cephalosporins belong to the $\beta$-lactam class of antibiotics and are presently the most commonly used antibiotics to treat gramnegative bacilli infection. E. coli strains can become resistant to beta lactam antibiotics by producing extended spectrum beta lactamase (ESBL), which is a plasmid-mediated $\beta$-lactamase that is capable of hydrolysing and inactivating $\beta$-lactams such as cephalosporins and monobactams (15). The identification of ESBL-producing E. coli (ESBL-E. coli) infections in infants in a neonatal intensive care unit is of particular concern because of the immature antibacterial immunity of neonates and the restricted therapeutic antibiotic options available (16).

The E. coli isolates often display resistance patterns that are typical of ESBL producers. In this study, the majority of the studied isolates showed resistance to amoxicillin (MIC range: $1.0->256 \mathrm{mg} / \mathrm{l}$ ), even though a very small proportion was resistant to a combination of amoxicillin with clavulanic acid (MIC range: 1.5-16). The antimicrobial resistance of experimental strains showed important differences between treatment with amoxicillin and amoxicillin-clavulanic acid. This indicates that while some E. coli strains can hydrolyze cephalosporins, this hydrolysis can be inhibited by clavulanic acid. A previous study had showed that these clinical E. coli isolates may produce group 2e $\beta$-lactamases (17). More recently, E. coli strains isolated from urine cultures of patients from Primary Care Barbastro Sector, between January 2011 and December 2013, showed a progressive increase to amoxicillinclavulanate that reached $21.5 \%$ in 2013 , a statistically significant increase (18) that was higher than the that presented in this study. Here, the E. coli strains isolated from the neonatal unit showed high resistance to amoxicillin, in accordance with the results found by Nitsch-Osuch et al. (19), which nevertheless described a relatively low degree of resistance to cephalosporins (1.8-5.3\%) and aminoglycosides $(0-2.6 \%)$ that were lower than our study. Bergin et al. (20) used multivariable logistic regression to evaluate the association between 30-day mortality and ampicillin-resistant E. coli bloodstream infections and were able to identify $123(48 \%)$ ampicillin-resistant isolates. However, the authors found no significant association between ampicillin resistance and increased mortality, nor between antibiotic therapy and lower mortality.

Furthermore, Monsef et al. (21) reported a higher resistance of $E$. coli cultured from neonatal patients to cephalosporins and aminoglycosides. In our study, most $E$. coli isolates were resistant to cefuroxime (65 out of 100, MIC range: 0.016$256 \mu \mathrm{g} / \mathrm{ml}$ ) and cefatriaxone (60 out of 100, MIC range: $0.002-$ $32 \mu \mathrm{g} / \mathrm{ml}$ ). Moreover, all isolates were susceptible to meropenem (MIC: $<0.002 \mu \mathrm{g} / \mathrm{ml}$ ), and the vast majority of the strains were susceptible to a combination of cefoperazone with clavulanic acid (MIC range: $<0.016-32 \mu \mathrm{g} / \mathrm{ml}$ ). We note that the majority of 
E. coli strains were also resistant to non- $\beta$-lactam antimicrobial agents, and some were resistant to sulfonamides (47 out of 100, MIC range: $0.002-32 \mu \mathrm{g} / \mathrm{ml}$ ), ciprofloxacin (33 out of 100 , MIC range: $0.002-32 \mu \mathrm{g} / \mathrm{ml}$ ), and gentamicin (20 out of 100 , MIC range: $0.016-256 \mu \mathrm{g} / \mathrm{ml}$ ). As previously reported (22), tigecycline demonstrates excellent activity against a wide variety of Gram-positive and Gram-negative bacteria, including ESBL-producing organisms, and should thus be considered an encouraging antimicrobial agent. However, this antibiotic is not recommended in younger patients ( $<18$ years of age) due to a lack of data regarding its safe usage, a problem that is potentially greater in the case of neonates (6).

Vernaz et al. (23) performed a retrospective observational time-series analysis to evaluate the incidence of nonduplicate clinical isolates of $E$. coli resistant to ciprofloxacin, trimethoprim/sulfamethoxazole and cefepime, from January 2000 through December 2007. The authors observed an increase in fluoroquinolone resistance among $\mathrm{CA}$ and HA isolates of E. coli, with slightly higher rates in the latter group, in accordance with data obtained from other European countries. They noted that the rate of ciprofloxacin resistance in E. coli is approaching the resistance rate of trimethoprim/sulfamethoxazole, and found that ciprofloxacin and cefepime resistance increased, Trimethoprim/sulfamethoxazole resistance remained stable, and total antibiotic use increased in both inpatient and outpatient settings. These results support efforts to reduce the prescription of fluoroquinolones for controlling resistant E. coli, including extended-spectrum $\beta$-lactamase producers.

In our study, we used meropenem as a treatment option to multidrug-resistant E. coli bacteremia. The recommended dosage of meropenem is calculated at $20 \mathrm{mg} / \mathrm{kg}$ q $8 \mathrm{~h}$ in neonates. Importantly, the use of meropenem in neonates warrants more concerns because of possible side effects such as anaphylaxis, liver and kidney impairment, and hemorrhagic symptoms. Even though all E. coli isolates from our study were sensitive to meropenem, it is not possible to fully evaluate the efficacy and safety of using carbapenems in pediatric patients, especially in neonates, and it is necessary to consider the existence of possible side effects. Meropenem and Imipenem are both members of carbapenems, a clinically important antibiotic family that is used in the treatment of Multidrug-Resistant (MDR) bacterial infections. However, susceptibility tests performed by the KirbyBauer disk diffusion method demonstrated that Imipenem sensitive E. coli BL21 cells overexpressing Ar-BVMO become resistant to this antibiotic. Agar disc diffusion assay further corroborates that, when Imipenem reacts with Ar-BVMO, it loses its antibiotic properties (24).

More than $25 \%$ of the isolates were resistant to at least three different classes of antibiotics in our study. A notable

\section{REFERENCES}

1. Harbeson D, Ben-Othman R, Amenyogbe N, Kollmann TR. Outgrowing the immaturity myth: the cost of defending from neonatal infectious disease. Front Immunol. (2018) 9:1077. doi: 10.3389/fimmu.2018. 01077 proportion showed cephalosporin resistant that probably reflects the epidemiology of ESBL-producing Enterobacteriaceae in China and the remainder of Asia.

Maternal treatment with antibiotics during pregnancy or at delivery should be considered as a possible influencing factor of $E$. coli neonatal resistance. In this study, three mothers had fungal vaginitis that was cured before pregnancy, so we expect this had no effect on the results observed here. In fact, we found a very close correlation between clinical manifestations and antibiotic resistance. All patients accepted our initial use of experimental anti-infection treatment, which was adjusted in the middle of the therapy according to the results obtained for the drug sensitivity test. All patients either improved or were cured, and were discharged from the hospital after successful therapeutic effects.

\section{CONCLUSION}

Drug-resistant E. coli has become an important and complex problem in clinical treatment. This work reports a high rate of antimicrobial resistance, including ESBL positivity and multidrug resistance in a set of $100 \mathrm{E}$. coli isolates from neonates admitted into a large NICU. The data reported here are highly relevant for local antimicrobial prescription practice. In the future, this research can be expanded to increase sample size and type, and to enable evaluation of the correlation between clinical manifestations and antibiotic resistance. This newly available information about E. coli resistance in newborns will help informing clinical evaluation and decision-making.

\section{DATA AVAILABILITY STATEMENT}

The raw data supporting the conclusions of this article will be made available by the authors, without undue reservation.

\section{AUTHOR CONTRIBUTIONS}

YW designed the study. DW collected the data, analyzed the data, and wrote the first draft of the manuscript, which was significantly edited by YW. YD, KY, and WG were participants in the workshop and the round-table and either gave presentations. All authors read and approved the final manuscript.

\section{FUNDING}

This work was funded by the National Natural Science Foundation of China (Grant No. 81872676) and Natural Science Foundation of Beijing Municipality (Beijing Natural Science Foundation) (Grant No. 7192063).
2. Liu L, Johnson HL, Cousens S, Perin J, Scott S, Lawn JE, et al. Global, regional, and national causes of child mortality: an updated systematic analysis for 2010 with time trends since 2000. Lancet. (2012) 379:215161. doi: 10.1016/S0140-6736(12)60560-1

3. Jones RN, Sader HS, Fritsche TR, Pottumarthy S. Comparisons of parenteral broad-spectrum cephalosporins tested against bacterial 
isolates from pediatric patients: report from the SENTRY antimicrobial surveillance program (1998-2004). Diagn Microbiol Infect Dis. (2007) 57:109-16. doi: 10.1016/j.diagmicrobio.2006.06.011

4. Tivendale KA, Logue CM, Kariyawasam S, Jordan D, Hussein A, Ganwu L, et al. Avian-pathogenic Escherichia coli strains are similar to neonatal meningitis $E$. coli strains and are able to cause meningitis in the rat model of human disease. Infect Immun. (2010) 78:3412-9. doi: 10.1128/IAI.00347-10

5. Loque CM, Doetkott C, Manqiamele P, Wannemuehler YM, Johnson TJ, Tivendale KA, et al. Genotypic and phenotypic traits that distinguish neonatal meningitis-associated Escherichia coli from fecal E. coli isolates of healthy human hosts. Appl Environ Microbiol. (2012) 78:5824-30. doi: 10.1128/AEM.07869-11

6. Franiczek R, Sobieszczanska B, Turniak M, Kasprzykowska U, Krzyzanowska B, Jermakow K, et al. ESBL-producing Escherichia coli isolated from children with acute diarrhea - antimicrobial susceptibility, adherence patterns and phylogenetic background. Adv Clin Exp Med. (2012) 21:187-92.

7. Clinical and Laboratory Standard Institute. Performance Standards for Antimicrobial Susceptibility Testing. Wayne PA: 18th Informational Supplement, M100-S18 (2008).

8. Cole BK, Ilikj M, McCloskey CB, Chavez-Bueno S. Antibiotic resistance and molecular characterization of bacteremia Escherichia coli isolates from newborns in the United States. PLoS ONE. (2019) 14:e0219352. doi: 10.1371/journal.pone.0219352

9. Powell EC, Mahajan PV, Roosevelt G, Hoyle JD Jr, Gattu R, Cruz AT, et al. Epidemiology of bacteremia in febrile infants aged 60 days and younger. Ann Emerg Med. (2018) 71:211-6. doi: 10.1016/j.annemergmed.2017.07.488

10. Mendoza-Palomar N, Balasch-Carulla M, Lauro SG, Cespedes MC, Andreu A, Frick MA, et al. Escherichia coli early-onset sepsis: trends over two decades. Eur J Pediatr. (2017) 176:1227-34. doi: 10.1007/s00431-017-2975-Z

11. Li JY, Chen SQ, Yan YY, Hu YY, Wei J, Wu QP, et al. Identification and antimicrobial resistance of pathogens in neonatal septicemia in China - A meta-analysis. Int J Infect Dis. (2018) 71:89-93. doi: 10.1016/j.ijid.2018.04.794

12. Zhu ML, Jin YT, Duan Y, He MZ, Lin ZL, Jing L. Multi-drug resistant Escherichia coli causing early-onset neonatal sepsis - a single center experience from China. Infect Drug Resist. (2019) 12:3695-702. doi: 10.2147/IDR.S229799

13. Sherchan JB, Hayakawa K, Miyoshi-Akiyama T, Ohmagari N, Kirikae $\mathrm{T}$, Nagamatsu $\mathrm{M}$, et al. Clinical epidemiology and molecular analysis of extended-spectrum B-lactamase-producing Escherichia coli in Nepal: characteristics of sequence types 131 and 648. Antimicrob Agents Chemother. (2015) 59:3424-32. doi: 10.1128/AAC.00270-15

14. Shlaes DM, Sahm D, Opiela C, Spellberg B. The FDA reboot of antibiotic development. Antimicrob Agents Chemother. (2013) 57:46057. doi: 10.1128/AAC.01277-13

15. Chmlelarczyk A, Wojkowska-Mach J, Romaniszyn D, Adamski P, Helwich E, Lauetrbach R, et al. Mode of delivery and other risk factors for Escherichia coli infections in very low birth weight infants. BMC Pediatr. (2014) 18:274. doi: 10.1186/1471-2431-14-274
16. Nakamura K, Kaneko M, Abe Y, Yamamoto N, Mori H, Yoshida A, et al. Outbreak of extended-spectrum $\beta$-lactamase-producing Escherichia coli transmitted through breast milk sharing in a neonatal intensive care unit. $J$ Hosp Infect. (2016) 92:42-6. doi: 10.1016/j.jhin.2015.05.002

17. Bush K, Jacoby GA. Updated functional classification of $\beta$-lactamases. Antimicrob Agents Chemother. (2010) 5:969-76. doi: 10.1128/AAC.01009-09

18. Betran A, Cortes AM, Lopez C. Evaluation of antibiotic resistance of Escherichia coli in urinary tract infections in primary care barbastro sector (Huesca). Rev Esp Quimioter. (2015) 28:263-6.

19. Nitsch-Osuch A, Choroszy-Krol I, Kuchar E, Korzeniewski K, Zycinska K, Wardyn K. Microbiological spectrum and susceptibility pattern of clinical isolates from the neonatal unit in a single medical center. Adv Clin Exp Med. (2015) 24:15-22. doi: 10.17219/acem/38170

20. Bergin SP, Thaden JT, Ericson JE, Cross H, Messina J, Clark RH, et al. Neonatal Escherichia coli bloodstream infections: clinical outcomes and impact of initial antibiotic therapy. Pediatr Infect Dis J. (2015) 34:9336. doi: 10.1097/INF.0000000000000769

21. Monsef A, Eghbaklian F. Antibiotic sensitivity pattern of common bacterial pathogens in NICU and neonatal ward in Hammedan province of Iran. Health. (2010) 2:625-9. doi: 10.4236/health.2010. 26094

22. Franiczek R, Krzyzanowska B, Dolna I, Mokracka-Latajka G. Conjugative transfer of plasmid-mediated CTX- M-type $\beta$-lactamases from clinical strains of Enterobacteriaceae to Salmonella enterica serovars. Adv Clin Exp Med. (2010) 19:313-22.

23. Vernaz N, Huttner B, Muscionico D, Salomon JL, Bonnabry P, LopezLozano JM, et al. Modelling the impact of antibiotic use on antibioticresistant Escherichia coli using population-based data from a large hospital and its surrounding community. J Antimicrob Chemother. (2011) 66:92835. doi: $10.1093 / \mathrm{jac} / \mathrm{dkq} 525$

24. Minerdi D, Zgrablic I, Castrignano S, Catucci G, Medana C, Terlizzi ME, et al. Escherichia coli overexpressing a Baeyer-Villiger monooxygenase from Acinetobacter radioresistens become resistant to Imipenem. Antimicrob Agents Chemother. (2016) 60:64-74. doi: 10.1128/AAC.0 1088-15

Conflict of Interest: The authors declare that the research was conducted in the absence of any commercial or financial relationships that could be construed as a potential conflict of interest.

Copyright (c) 2021 Wu, Ding, Yao, Gao and Wang. This is an open-access article distributed under the terms of the Creative Commons Attribution License (CC BY). The use, distribution or reproduction in other forums is permitted, provided the original author(s) and the copyright owner(s) are credited and that the original publication in this journal is cited, in accordance with accepted academic practice. No use, distribution or reproduction is permitted which does not comply with these terms. 